NASA Technical Memorandum 106209 ICOMP-93-19

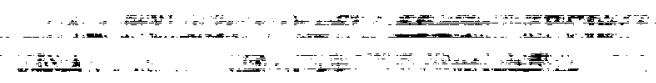

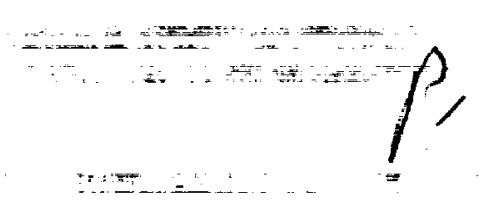

$22=$

\title{
Accuracy of Least-Squares Methods For The Navier-Stokes Equations
}

Pavel B. Bochev

Virginia Polytechnic Institute and State University

Blacksburg, Virginia

and

Max D. Gunzburger

Institute for Computational Mechanics in Propulsion

Lewis Research Center

Cleveland, Ohio

and Virginia Polytechnic Institute and State University

Blacksburg, Virginia

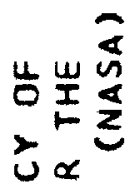

$\$$

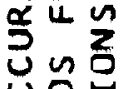

은

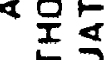

至号

o

는

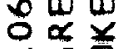

존은

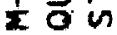

1 i 1

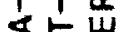

nु

व $4>$

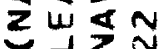

June 1993 
- 


\title{
ACCURACY OF LEAST-SQUARES METHODS FOR THE NAVIER-STOKES EQUATIONS ${ }^{*}$
}

\author{
Pavel B. Bochev \\ Virginia Polytechnic Institute and State University \\ Blacksburg, Virginia 24061-0531 \\ and \\ Max D. Gunzburger \\ Institute for Computational Mechanics in Propulsion \\ Lewis Research Center \\ Cleveland, Ohio 44135 \\ and Virginia Polytechnic Institute and State University \\ Blacksburg, VA 24061-0531
}

\begin{abstract}
Recently there has been substantial interest in least-squares finite element methods for velocity-vorticitypressure formulations of the incompressible Navier-Stokes equations. The main cause for this interest is the fact that algorithms for the resulting discrete equations can be devised which require the soluiton of only symmetric, positive definite systems of algebraic equations. On the other hand, it is well-documented that methods using the vorticity as a primary variable often yield very poor approximations. Thus, here we study the accuracy of these methods through a series of computational experiments, and also comment on theoretical error estimates. It is found, despite the failure of standard methods for deriving error estimates, that computational evidence suggests that these methods are, at the least, nearly optimally accurate. Thus, in addition to the desirable matrix properties yielded by least-squares methods, one also obtains accurate approximations.
\end{abstract}

\section{INTRODUCTION}

The approximate solution of the Navier-Stokes equations of incompressible flow has received tremendous attention from engineers and mathematicians; see, e.g., [9], [10], or [11]. Among the more recent developments has been the use of least-squares ideas; see, e.g., [7] for a recent survey of one such approach. Also, truly least-squares methods have been developed and applied; see, e.g., [3]-[5], [13]-[17], [20], and [22].

Here, a finite element method based on a least-squares variational principle is examined for the approximate solution of the stationary, incompressible Navier-Stokes equations. These equations are cast into a first-order system of partial differential equations involving the velocity, vorticity, and pressure as dependent variables. In three-dimensions one has seven unknown scalar fields. However, the application of a least-squares principle along with, for example, a Newton linearization, results in symmetric, positive definite linear systems, at least in a neighborhood of the solution. The influence of the Reynolds number on the positive definiteness of these linear systems is felt only through the size of the neighborhood. Thus, if properly implemented continuation (with respect to the Reynolds number) methods are used, one can expect to only encounter symmetric, positive definite linear systems in the solution procedure. A further advantage of this method is that a single piecewise polynomial finite element space may be used for all test and trial functions, i.e., one may use equal

\footnotetext{
"This work was supported in part by the Air Force Office of Scientific Research under grant number AFOSR-90-0179. MDG was also supported by the Institute for Computational Mechanics in Propul sion at the NASA Lewis Research Center. Work funded under NASA Cooperative Agreement NCC3-233.
} 
order interpolation with respect to a single grid for all dependent variables and test functions. A final advantage resulting from the use of a least-squares principle is that, unlike some other methods involving the vorticity, no artificial numerical boundary conditions for the vorticity need be devised.

The method discussed here is similar to the ones of [3]-[5] and [13]-[17]; however, there are certain differences as well, especially in the order in which the least-squares, the discretization, and the linearizations steps are taken. Furthermore, the analyses found in some of these papers are incorrect, leaving open the question of the accuracy of approximations.

In $\S 2$, we define the least-squares finite element method. In $\S 3$, we discuss some practical and theoretical issues connected with the method described in $\S 2$. Then, in $\S 4$, we give the results of a computational study of the accuracy of the algorithm. Finally, in $\S 5$ we give some concluding remarks.

\section{THE LEAST-SQUARES FINITE ELEMENT METHOD}

\section{1 - The velocity-vorticity-pressure equations}

Let the bounded set $\Omega \subset \mathbf{R}^{3}$ denote the flow domain and let $\Gamma$ denote its boundary. The dimensionless equations governing the steady incompressible flow of a viscous fluid may be written in the form

$$
\begin{gathered}
\operatorname{div} \mathbf{u}=0 \text { in } \Omega, \\
\operatorname{curl} \mathbf{u}-\boldsymbol{\omega}=0 \text { in } \Omega, \\
\nu \operatorname{curl} \omega+\boldsymbol{\omega} \times \mathbf{u}+\operatorname{grad} p=\mathbf{f} \text { in } \Omega,
\end{gathered}
$$

and

$$
\operatorname{div} \omega=0 \text { in } \Omega \text {, }
$$

where $u, p$, and $\boldsymbol{\omega}$ denote the velocity, pressure, and vorticity fields, respectively, $\nu$ the inverse of the Reynolds number, and $f$ a given body force. The first-order system of partial differential equations (2.1)-(2.4) constitutes the velocity-vorticity-pressure form of the equations of steady, incompressible flow. Note that the terminology "pressure" is a little misleading since the variable $p$ is actually the total head, i.e., $p=\hat{p}+\frac{1}{2}|\mathbf{u}|^{2}$, where $\hat{p}$ denotes the pressure.

In view of (2.2), it seeems that (2.4) is redundant. However, it is crucial to the stability of the least-squares algorithm to explicitly require (2.4); see, e.g., [3] and [15].

The system (2.1)-(2.4) should be supplemented with boundary conditions. Here we examine two such boundary conditions. The first is to impose the velocity on the boundary, i.e.,

$$
\mathrm{u}=\mathbf{U}_{1} \text { on } \Gamma \text {, }
$$


where $U_{1}$ denotes a given function defined along $\Gamma$. Note that (2.5) implies that the normal component of the vorticity is also known, i.e.,

$$
\boldsymbol{\omega} \cdot \mathbf{n}=\mathbf{n} \cdot \operatorname{curl} \mathbf{U}_{1} \text { on } \Gamma
$$

where $\mathbf{n}$ denotes the unit outer normal to $\Omega$. To see this, one merely needs to observe that $\mathbf{n} \cdot \operatorname{curl} \mathbf{u}$ involves only tangential derivatives of the tangential components of $\mathbf{u}$ and these may be deduced from (2.5). To these one must add a condition to fix the pressure; we choose to fix the pressure at a single point $x_{0}$ in $\bar{\Omega}$, i.e.,

$$
p\left(\mathbf{x}_{0}\right)=P_{0},
$$

where $P_{0}$ is a given number.

The second combination of boundary conditions we consider is the pressure and the normal component of the velocity, i.e.,

$$
p=P \text { on } \Gamma
$$

and

$$
\mathbf{u} \cdot \mathbf{n}=U_{2} \text { on } \Gamma
$$

where $P$ and $U_{2}$ denote given functions defined along $\Gamma$. The boundary conditions (2.8)-(2.9) are not all that useful as boundary conditions for the Navier-Stokes equations; we consider them here for two reasons. First, a complete, rigorous analysis of least-squares finite element approximations of (2.1)-(2.4) and (2.8)-(2.9) can be given using standard techniques; this is not the case for (2.5)-(2.7). Furthermore (2.1)-(2.4) and (2.8)-(2.9) can be shown to be related to second order elliptic partial differential equations; we will discuss these issues in more detail below.

\section{2 - The least-squares principle}

One can use (2.1)-(2.4) to define the least-squares functional

$$
\begin{aligned}
\mathcal{J}(\mathbf{u}, \boldsymbol{\omega}, p)=\int_{\Omega}\left(|\operatorname{curl} \mathbf{u}-\boldsymbol{\omega}|^{2}\right. & +|\operatorname{div} \mathbf{u}|^{2}+|\operatorname{div} \boldsymbol{\omega}|^{2} \\
& \left.+|\nu \operatorname{curl} \boldsymbol{\omega}+\boldsymbol{\omega} \times \mathbf{u}+\operatorname{grad} p-\mathbf{f}|^{2}\right) d \Omega .
\end{aligned}
$$

The least-squares principle then requires the minimization of this functional over appropriate function spaces. Then standard techniques from the calculus of variations may be used to deduce that minimzers $(\mathbf{u}, p, \boldsymbol{\omega})$ of $\mathcal{J}$ necessarily satisfty

$$
\begin{aligned}
\int_{\Omega}[(\operatorname{curl} \mathbf{u}-\boldsymbol{\omega}) \cdot \operatorname{curl} \mathbf{v}+\operatorname{div} \mathbf{u} \operatorname{div} \mathbf{v} \\
+(\nu \operatorname{curl} \omega+\operatorname{grad} p+\boldsymbol{\omega} \times \mathbf{u}-\mathbf{f}) \cdot(\boldsymbol{\omega} \times \mathbf{v})] d \Omega=0
\end{aligned}
$$




$$
\begin{aligned}
\int_{\Omega}[\operatorname{div} \boldsymbol{\omega} \operatorname{div} \boldsymbol{\zeta}-(\operatorname{curl} \mathbf{u}-\boldsymbol{\omega}) \cdot \boldsymbol{\zeta} & \\
& +(\nu \operatorname{curl} \boldsymbol{\omega}+\operatorname{grad} p+\boldsymbol{\omega} \times \mathbf{u}-\mathbf{f}) \cdot(\nu \operatorname{curl} \boldsymbol{\zeta}+\boldsymbol{\zeta} \times \mathbf{u})] d \Omega=0
\end{aligned}
$$

and

$$
\int_{\Omega}(\nu \operatorname{curl} \omega+\operatorname{grad} p+\omega \times \mathbf{u}-\mathbf{f}) \cdot \operatorname{grad} q d \Omega=0 .
$$

In (2.11)-(2.13) the test functions $(v, q, \zeta)$ are required to belong to suitable function spaces; we do not go into detail here since we are primarily interested in finite element discretizations of these equations.

Clearly any solution $(\mathbf{u}, \omega, p)$ of, say $(2.1)-(2.7)$, satisfies (2.11)-(2.13).

\section{3 - The two-dimensional case}

For planar flows we have that $\mathbf{u}=\left(u_{1}, u_{2}, 0\right)^{T}$ and $u_{1}, u_{2}$, and $p$ are functions of $x_{1}$ and $x_{2}$ only. Then, we have that $\omega=(0,0, \omega)^{T}$ where $\omega=\partial u_{2} / \partial x_{1}-\partial u_{1} / \partial x_{2}$. In this case, the system (2.1)-(2.4) simplifies to

$$
\frac{\partial u_{1}}{\partial x_{1}}+\frac{\partial u_{2}}{\partial x_{2}}=0 \text { in } \Omega
$$$$
\frac{\partial u_{2}}{\partial x_{1}}-\frac{\partial u_{1}}{\partial x_{2}}-\omega=0 \text { in } \Omega
$$

$$
\nu \frac{\partial \omega}{\partial x_{2}}+\frac{\partial p}{\partial x_{1}}-u_{2} \omega=f_{1} \text { in } \Omega
$$

and

$$
-\nu \frac{\partial \omega}{\partial x_{1}}+\frac{\partial p}{\partial x_{2}}+u_{1} \omega=f_{2} \text { in } \Omega
$$

where $\mathbf{f}=\left(f_{1}, f_{2}, 0\right)^{T}$. The boundary conditions (2.5)-(2.7) reduce to just (2.5) and (2.7) and the boundary conditions (2.8)-(2.9) remain unchanged. The functional (2.10) and the necessary conditions (2.11)-(2.13) also simplify in the obvious manner for two-dimensional problems.

\section{4 - Finite element methods}

Starting with the weak formulation (2.11)-(2.13), a conforming finite element method can be defined in a completely standard manner. We choose a finite element space $S^{h}$ parametrized by $h$. For example, for a given positive integer $r, S^{h}$ could consist of continuous (over $\Omega$ ) piecewise polynomials of degree less than or equal to $r$ with respect to a subdivision of $\Omega$ into finite elements. In this case the parameter $h$ may be related to the size of the grid. We then define the spaces $\mathbf{S}^{h}=\left\{\mathbf{v} \mid v_{i} \in S^{h}, i=1,2,3\right\}$,

$$
\mathbf{V}_{0}^{h}=\left\{\mathbf{v} \in \mathbf{S}^{h} \mid \mathbf{v}=\mathbf{0} \text { on } \Gamma\right\},
$$




$$
\mathbf{Z}_{0}^{h}=\left\{\zeta \in \mathbf{S}^{h} \mid \zeta \cdot \mathbf{n}=0 \text { on } \Gamma\right\}
$$

and

$$
Q_{0}^{h}=\left\{q \in S^{h} \mid q\left(\mathbf{x}_{0}\right)=0\right\} .
$$

For the boundary conditions (2.5)-(2.7), the discrete problem is defined as follows: seek $\mathbf{u}^{h} \in \mathbf{S}^{h}$, $\boldsymbol{\omega}^{h} \in \mathbf{S}^{h}$, and $p^{h} \in S^{h}$ such that $\mathbf{u}^{h}=\mathrm{U}_{1}^{h}$ and $\omega^{h} \cdot \mathbf{n}=W^{h}$ on $\Gamma, p^{h}\left(\mathbf{x}_{0}\right)=P_{0}$,

$$
\begin{aligned}
& \int_{\Omega}\left[\left(\operatorname{curl} \mathbf{u}^{h}-\omega^{h}\right) \cdot \operatorname{curl} \mathbf{v}^{h}+\operatorname{div} \mathbf{u}^{h} \operatorname{div} \mathbf{v}^{h}\right. \\
& \left.\quad+\left(\nu \operatorname{curl} \omega^{h}+\operatorname{grad} p^{h}+\omega^{h} \times \mathbf{u}^{h}-\mathbf{f}\right) \cdot\left(\omega^{h} \times \mathbf{v}^{h}\right)\right] d \Omega=0 \quad \forall \mathbf{v}^{h} \in \mathbf{V}_{0}^{h}, \\
& \int_{\Omega}\left[\operatorname{div} \omega^{h} \operatorname{div} \zeta^{h}-\left(\operatorname{curl} \mathbf{u}^{h}-\omega^{h}\right) \cdot \zeta^{h}\right. \\
& \left.\quad+\left(\nu \operatorname{curl} \omega^{h}+\operatorname{grad} p^{h}+\omega^{h} \times \mathbf{u}^{h}-\mathbf{f}\right) \cdot\left(\nu \operatorname{curl} \zeta^{h}+\zeta^{h} \times \mathbf{u}^{h}\right)\right] d \Omega=0 \quad \forall \zeta^{h} \in \mathbf{Z}_{0}^{h},
\end{aligned}
$$

and

$$
\int_{\Omega}\left(\nu \operatorname{curl} \omega^{h}+\operatorname{grad} p^{h}+\omega^{h} \times \mathbf{u}^{h}-\mathbf{f}\right) \cdot \operatorname{grad} q^{h} d \Omega=0 \quad \forall q^{h} \in Q_{0}^{h}
$$

are satisfied. Here $\mathbf{U}_{1}^{h}$ and $W^{h}$ are approximations of the data $U_{1}$ and curl $U_{1} \cdot \mathbf{n}$, respectively. For example, we could define the former pair to be boundary interpolants of the latter pair.

Note that all of the discrete variables, i.e., $q^{h}$ and the components of $\mathbf{u}^{h}$ and $\boldsymbol{\omega}^{h}$, are approximated by the same degree continuous piecewise polymomials defined with respect to a single grid.

The discrete problem for the boundary conditions (2.8)-(2.9) is also given by (2.18)-(2.20) except that now we have that $\mathbf{u}^{h} \cdot \mathbf{n}=U_{2}^{h}$ and $p^{h}=P^{h}$ on $\Gamma$ and the test functions spaces in (2.18)-(2.20) must be suitably redefined to account for the boundary conditions $\mathbf{v}^{h} \cdot \mathbf{n}=0$ and $q^{h}=0$ on $\Gamma$. Again, $U_{2}^{h}$ and $P^{h}$ are approximations to $U_{2}$ and $P$, respectively.

\section{PRACTICAL AND THEORETICAL ISSUES}

\section{1 - Newton's method}

The discrete equations (2.18)-(2.20) are a nonlinear system of algebraic equations that must be solved in an iterative manner. There are many methods that one might use for such a purpose; here we only consider Newton's method. However, we do remark that if a quasi-Newton method is used, it should be chosen so that it preserves symmetry and positive definiteness of the approximate Hessian matrices.

Newton's method for the solution of (2.18)-(2.20) is defined as follows. Given initial guesses $\mathbf{u}^{(0)}$, $\boldsymbol{\omega}^{(0)}$, and $p^{(0)}$ for $\mathbf{u}^{h}, \boldsymbol{\omega}^{h}$, and $p^{h}$, respectively, the sequence of Newton iterates $\left\{\mathbf{u}^{(k)}, \boldsymbol{\omega}^{(k)}, p^{(k)}\right\}_{k>0}$ 
is generated recursively by solving, for $k=1,2, \ldots$, the system

$$
\begin{aligned}
& \int_{\Omega}[\left(\operatorname{curl} \mathbf{u}^{(k)}-\omega^{(k)}\right) \cdot \operatorname{curl} \mathbf{v}^{h}+\operatorname{div} \mathbf{u}^{(k)} \operatorname{div} \mathbf{v}^{h} \\
&+\left(\nu \operatorname{curl} \omega^{(k)}+\operatorname{grad} p^{(k)}+\omega^{(k)} \times \mathbf{u}^{(k-1)}+\omega^{(k-1)} \times \mathbf{u}^{(k)}\right) \cdot\left(\omega^{(k-1)} \times \mathbf{v}^{h}\right) \\
&\left.+\left(\nu \operatorname{curl} \omega^{(k-1)}+\operatorname{grad} p^{(k-1)}+\omega^{(k-1)} \times \mathbf{u}^{(k-1)}-\mathbf{f}\right) \cdot\left(\omega^{(k)} \times \mathbf{v}^{h}\right)\right] d \Omega \\
&= \int_{\Omega}\left(\nu \operatorname{curl} \omega^{(k-1)}+\operatorname{grad} p^{(k-1)}+2 \omega^{(k-1)} \times \mathbf{u}^{(k-1)}\right) \cdot\left(\omega^{(k-1)} \times \mathbf{v}^{h}\right) d \Omega \forall \mathbf{v}^{h} \in \mathbf{v}_{0}^{h}, \\
& \int_{\Omega}\left[\left(\operatorname{div} \omega^{(k)} \operatorname{div} \zeta^{h}-\left(\operatorname{curl} \mathbf{u}^{(k)}-\omega^{(k)}\right) \cdot \zeta^{h}\right.\right. \\
& \quad+\left(\nu \operatorname{curl} \omega^{(k)}+\operatorname{grad} p^{(k)}+\omega^{(k)} \times \mathbf{u}^{(k-1)}+\omega^{(k-1)} \times \mathbf{u}^{(k)}\right) \cdot\left(\nu \operatorname{curl} \zeta^{h}\right) \\
& \quad+\left(\nu \operatorname{curl} \omega^{(k)}+\operatorname{grad} p^{(k)}+\omega^{(k)} \times \mathbf{u}^{(k-1)}+\omega^{(k-1)} \times \mathbf{u}^{(k)}\right) \cdot\left(\zeta^{h} \times \mathbf{u}^{(k-1)}\right) \\
&\left.\quad+\left(\nu \operatorname{curl} \omega^{(k-1)}+\operatorname{grad} p^{(k-1)}+\omega^{(k-1)} \times \mathbf{u}^{(k-1)}-\mathbf{f}\right) \cdot\left(\zeta^{h} \times \mathbf{u}^{(k)}\right)\right] d \Omega \\
&= \int_{\Omega}\left[\nu\left(\mathbf{f}+\omega^{(k-1)} \times \mathbf{u}^{(k-1)}\right) \cdot \operatorname{curl} \zeta^{h}\right. \\
&\left.+\left(\nu \operatorname{curl} \omega^{(k-1)}+\operatorname{grad} p^{(k-1)}+2 \omega^{(k-1)} \times \mathbf{u}^{(k-1)}\right) \cdot\left(\zeta^{h} \times \mathbf{u}^{(k-1)}\right)\right] d \Omega \quad \forall \zeta^{h} \in \mathbf{Z}_{0}^{h},
\end{aligned}
$$

and

$$
\begin{gathered}
\int_{\Omega}\left(\nu \operatorname{curl} \omega^{(k)}+\operatorname{grad} p^{(k)}+\omega^{(k)} \times \mathbf{u}^{(k-1)}+\omega^{(k-1)} \times \mathbf{u}^{(k)}\right) \cdot \operatorname{grad} q^{h} d \Omega \\
=\int_{\Omega}\left(\mathrm{f}+\omega^{(k-1)} \times \mathbf{u}^{(k-1)}\right) \cdot \operatorname{grad} q^{h} d \Omega \quad \forall q^{h} \in Q_{0}^{h} .
\end{gathered}
$$

The system of linear algebraic equations (3.1)-(3.3) that is used to determine the $k$-th Newton iterate from the $(k-1)$-st looks rather formidable. However, it also has some very good features. First, it is easy to see that this system is symmetric. Moreover, in a neighborhood of a minimizer, the Hessian matrix for the functional of $(2.10)$ is necessarily positive definite; but this Hessian matrix is exactly the coefficient matrix of (3.1)-(3.3). Thus, in a neighborhood of a solution of (2.18)-(2.20), the system (3.1)-(3.3) is symmetric and positive definite. This feature is independent of the value of $\nu$, i.e., of the value of the Reynolds number. These observations, along with the guaranteed local and quadratic convergence of Newton's method, are potentially very advantageous.

\section{2 - Continuation methods}

At this point one may well ask what goes wrong with the method as the Reynolds number increases? Surely one difficulty is that the attraction ball for Newton's method, or for any other iterative method for solving the nonlinear equations, decreases in size with increasing Reynolds number. This is known to be true for other discretizations of the Navier-Stokes equations. A related observation is that the positive definiteness of the Hessian matrix is guaranteed only in a 
neighborhood of a minimizer; again the size of this neighborhood surely decreases with increasing Reynolds number. As a result, for an arbitrary initial guess we may have that Newton's method doesn't converge and/or that the coefficient matrix in (3.1)-(3.3) is not positive definite. The former is, of course, unacceptable, while the latter occurance would preclude the use of simple iterative methods for solving the linear systems (3.1)-(3.3) that define the Newton iterates.

In order to determine an initial guess that is within the attraction ball of Newton's method, and also is such that the coefficient matrix in (3.1)-(3.3) with $k=1$ is positive definite, one can use continuation or homotopy methods, among others. Here we describe a simple continuation method; see, e.g., [18], [19], or [21]. Let us symbolically express the system (2.18)-(2.20) in the form

$$
F\left(\mathbf{u}^{h}, \omega^{h}, p^{h} ; R e\right)=0 .
$$

Here $R e=1 / \nu$ denotes the target Reynolds number, i.e., the value of the Reynolds number at which we want a solution. Now, suppose we have a sequence of increasing Reynolds numbers $\left\{R e_{m}\right\}_{m=1}^{M}$ with $R e_{M}=R e$. We denote the solution of $(2.18)-(2.20)$ for $\nu_{m}=1 / R e_{m}$ by $\left(u_{m}, \omega_{m}, p_{m}\right)$. For any value of $m$, we obtain $\left(u_{m}, \omega_{m}, p_{m}\right)$ by Newton's method, i.e., we solve the sequence of linear systems

$$
\begin{aligned}
F^{\prime}\left(\mathrm{u}_{m}^{(k-1)}, \omega_{m}^{(k-1)}, p_{m}^{(k-1)} ; R e_{m}\right) & \cdot\left(\left(\mathbf{u}_{m}^{(k)}, \omega_{m}^{(k)}, p_{m}^{(k)}\right)-\left(\mathbf{u}_{m}^{(k-1)}, \omega_{m}^{(k-1)}, p_{m}^{(k-1)}\right)\right) \\
& =-F\left(\mathbf{u}_{m}^{(k-1)}, \omega_{m}^{(k-1)}, p_{m}^{(k-1)} ; R e_{m}\right) \quad \text { for } k=1,2, \ldots
\end{aligned}
$$

Here, $F^{\prime}$ denotes the Jacobian of $F$ with respect to $\left(\mathbf{u}^{h}, \boldsymbol{\omega}^{h}, p^{h}\right)$. We need to specify the initial guesses $\left(u_{m}^{(0)}, \omega_{m}^{(0)}, p_{m}^{(0)}\right)$ to be used to start, for each $m$, the iteration with respect to $k$ in (3.4). Assume that $R e_{1}$ is sufficiently small so that the iteration (3.4) converges if $\left(\mathbf{u}_{1}^{(0)}, \omega_{1}^{(0)}, p_{1}^{(0)}\right)$ is chosen to be the solution of a discrete linear Stokes problem. For example, we could choose $R e_{1}=1$ and take for $\left(u_{1}^{(0)}, \omega_{1}^{(0)}, p_{1}^{(0)}\right)$ the solution of (2.18)-(2.20) with all cross product terms deleted and with $\nu=1$. The latter problem involves a symmetric, positive definite linear algebraic system. The remaining initial guesses $\left\{\left(\mathbf{u}_{m}^{(0)}, \omega_{m}^{(0)}, p_{m}^{(0)}\right)\right\}_{m=2}^{M}$ are determined by "continuing along the tangent", i.e., by solving the linear algebraic system

$$
\begin{aligned}
F^{\prime}\left(\left(\mathbf{u}_{m-1}, \omega_{m-1}, p_{m-1} ; R e_{m-1}\right)\right. & \cdot\left(\left(\mathbf{u}_{m}^{(0)}, \omega_{m}^{(0)}, p_{m}^{(0)}\right)-\left(\mathbf{u}_{m-1}, \omega_{m-1}, p_{m-1}\right)\right) \\
= & -\left(R e_{m}-R e_{m-1}\right) F_{R e}\left(\left(\mathbf{u}_{m-1}, \omega_{m-1}, p_{m-1} ; R e_{m-1}\right) .\right.
\end{aligned}
$$

Here, $\left(\mathbf{u}_{m-1}, \boldsymbol{\omega}_{m-1}, p_{m-1}\right)$ denotes the converged iterates determined from (3.4) at the $(m-1)$-st stage and $F_{R e}$ denotes the Frechet derivative of $F$ with respect to the parameter $R e$. Note that the coefficient matrix of the linear system (3.5) may be chosen to be the same as the coefficient matrix for the last iteration of $(3.4)$ at the $(m-1)$-st stage.

The combined Newton-continuation method is now completely defined. If $\left(R e_{m}-R e_{m-1}\right)$ is sufficiently small, the use of (3.5) should yield initial guesses that are within the attraction ball of 
Newton's method and such that the coefficient matrices in (3.4) with $k=1$ are positive definite. (They are always symmetric, of course.) In fact, since Newton's method is guaranteed to be locally convergent, i.e., its attraction ball is nontrivial, and since the neighboorhood of a minimizer for which the Hessian matrix is positive definite is also nontrivial, by choosing $\left(R e_{m}-R e_{m-1}\right)$ sufficiently small, one can guarantee that the combined Newton-continuation method should only deal with symmetric, positive definite matrices.

The method can be made self-correcting. For example, suppose that the linear systems in (3.4) are solved by an iterative method, e.g., the conjugate gradient method, that works, or works well, only for symmetric positive definite matrices. Then, if we have chosen an increment $\left(R e_{m}-R e_{m-1}\right)$ that is too large, then either the Newton iteration or the conjugate gradient iteration will fail. In either case, one can restart the $m$-th stage by choosing a smaller value of $R e_{m}$ in (3.4) and (3.5).

We note that often the even simpler "continuation along a constant" method

$$
\left(u_{m}^{(0)}, \omega_{m}^{(0)}, p_{m}^{(0)}\right)=\left(\mathbf{u}_{m-1}, \omega_{m-1}, p_{m-1}\right)
$$

for generating initial guesses has been found to work well in viscous flow calculations; see [12]. The disadvantage of (3.6) it that it breaks down in the vicinity of bifurcation or turning points, while (3.5) can be ammended so that it can handle such singular points; see [18], [19], and [21].

We also note that there are modifications possible to Newton's method that circumvent the difficulty of not having positive definite Hessian matrices. For example, one simple such modification is to add to the diagonal entries of the coefficient matrix in (3.1)-(3.3) a multiple of the magnitude of the residual of the previous Newton iterate. In the notation of (3.4), we would replace the Jacobian matrix $F^{\prime}$ on the left-hand side with

$$
F^{\prime}\left(\mathbf{u}_{m}^{(k-1)}, \omega_{m}^{(k-1)}, p_{m}^{(k-1)} ; R e_{m}\right)+\gamma\left\|F\left(\mathbf{u}_{m}^{(k-1)}, \omega_{m}^{(k-1)}, p_{m}^{(k-1)} ; R e_{m}\right)\right\|
$$

where $\|\cdot\|$ denotes the Euclidean length. By choosing the constant $\gamma$ sufficietnly large we can make sure that the matrix represented by (3.7) is positive definite. However, as we approach the solution of the problem, the term multiplying $\gamma$ approaches zero so that we recover the local convergence properties of Newton's method in the neighborhood of the solution.

\section{3 - Enhancing mass conservation}

In many instances one is especially interested in conserving mass, i.e., satisfying the continuity equation (2.1) as well as possible. The method discussed here is not exactly mass conserving, i.e., $\operatorname{div} \mathbf{u}^{h} \neq 0$ exactly. In fact, one can easily show that at best $\operatorname{div} \mathbf{u}^{h} \approx C h^{r}$ whenever the finite element space, restricted to any element, contains all polynomials of degree less than or equal to $r$. We can reduce the size of the constant $C$ by introducing a weight into the functional (2.10). Indeed, if we replace the $|\operatorname{div} u|^{2}$ term in $(2.10)$ by $\alpha|\operatorname{div} \mathbf{u}|^{2}$, where $\alpha>0$ is a constant, then the constant $C$ can be shown to be proportional to $1 / \sqrt{\alpha}$. Thus, by choosing a large value of $\alpha$, one can make $\operatorname{div} \mathbf{u}^{h}$ small. This is another potential advantage of the least-squares method. However, one must 
keep in mind that the larger the value of $\alpha$, the worse one satisfies the momentum equation relative to the continuity equation.

\section{4 - Theoretical observations concerning accuracy}

Error estimates for the least-squares finite element approximations are derived through the following process. First, using the theory of [2] (see also [6] and [9]), one can show that the error estimates for the nonlinear Navier-Stokes equations are essentially the same as those for the linear Stokes problem, at least away from singular points. Now for the Stokes problem

$$
\begin{gathered}
\operatorname{div} \mathbf{u}=0 \text { in } \Omega, \\
\operatorname{curl} \mathbf{u}-\boldsymbol{\omega}=0 \text { in } \Omega, \\
\nu \operatorname{curl} \omega+\operatorname{grad} p=\mathrm{f} \text { in } \Omega,
\end{gathered}
$$

and

$$
\operatorname{div} \omega=0 \text { in } \Omega
$$

standard techniques for estimating the error of least-squares finite element approximations require that this system be elliptic and that the boundary conditions satisfy the complementing condition of [1]; see, e.g., [4], [5], and [23]. Moreover, in order to use finite element functions that are merely continuous across element boundaries, one must be able to bound the $L^{2}$-norm of the derivatives of solutions of (3.8)-(3.11) in terms of the data of the problem. For the boundary conditions (2.5)(2.7) this program cannot be carried out, i.e., the system (3.8)-(3.11) and (2.5)-(2.7) does not satisfy the complementing conditions of [1] when one requires that the derivatives of $u, \omega$, and $p$ be merely square integrable. Thus, standard error estimation techniques for least-squares finite element discretizations of (2.1)-(2.7) cannot be used.

On the other hand, one can easily show that the system (3.8)-(3.11) and (2.8)-(2.9) does satisfy all the requisite conditions of the theory of [1] and therefore one may conclude that in that case one may obtain optimal error estimates. For example, if continuous piecewise polynomials of degree $\leq r$ are used with respect to a triangulation of $\Omega$, then one can ultimately conclude that for sufficiently smooth solutions

$$
\left|\mathbf{u}-\mathbf{u}^{h}\right|_{1}+\left|\omega-\omega^{h}\right|_{1}+\left|p-p^{h}\right|_{1} \leq C h^{r}
$$

and, under mild restrictions on the domain,

$$
\left\|\mathbf{u}-\mathbf{u}^{h}\right\|_{0}+\left\|\boldsymbol{\omega}-\boldsymbol{\omega}^{h}\right\|_{0}+\left\|p-p^{h}\right\|_{0} \leq C h^{r+1},
$$


where $h$ denotes a measure of the grid size and where $\|\cdot\|_{0}$ and $|\cdot|_{1}$ denote the $L^{2}(\Omega)$-norm and the $L^{2}(\Omega)$-norm of the first derivatives, respectively.

Nothing we have said necessarily means that least-squares finite element approximations of (2.1)-(2.7) do not satisfy (3.12)-(3.13) as well. All we have concluded so far is that we cannot, using standard estimation techniques, derive these estimates. Indeed, this is the motivation for the computational study of $\S 4$.

\section{5 - Connection of (2.8)-(2.9) with second-order elliptic problems}

Consider the problem

$$
\Delta p=g \text { in } \Omega
$$

where $\Delta$ denotes the Laplacian operator, and

$$
p=P \text { on } \Gamma
$$

Now, let

$$
\mathbf{u}=\operatorname{grad} p \text { in } \Omega \text {. }
$$

Then we have that

$$
\operatorname{div} u=g \text { in } \Omega
$$

Thus (3.16)-(3.17) is a first-order system equivalent to (3.14). However, it can be shown that this first-order system is not elliptic and that in general least-squares finite element methods for (3.16)(3.17) are not stable; see [8]. However, if one considers the system (3.17),

$$
\text { curl } \omega+\operatorname{grad} p=\mathbf{u}, \quad \operatorname{curl} \mathbf{u}=\mathbf{0} \text { and } \operatorname{div} \omega=0 \text { in } \Omega,
$$

then it can be shown that this sytem is elliptic. Clearly, if $p$ is a solution of (3.17)-(3.18) and (3.15), then $p$ is also a solution of (3.14)-(3.15). Note that the principal part of (3.17)-(3.18), i.e., the differentiated terms, are identical to those of (2.1)-(2.4), and that the boundary condition (3.15) is the same as (2.8). We can then infer the optimal accuracy of least-squares finite element methods for appropriately defined first-order systems arising from second-order ellliptic equations.

\section{A COMPUTATIONAL STUDY OF ACCURACY}

The accuracy of least-squares finite element approximations for the Navier-Stokes equations are essentially the same as that for the linear Stokes equations. Moreover, the accuracy is independent of dimension. Thus, here we restrict attention to the Stokes equations in two-dimensions. We also 
take for our domain the unit square $\Omega=\{0 \leq x \leq 1,0 \leq y \leq 1\}$. Thus, we consider the generalized Stokes equations

$$
\frac{\partial u}{\partial x}+\frac{\partial v}{\partial y}=g_{1} \quad \text { in } \Omega
$$

$$
\frac{\partial v}{\partial x}-\frac{\partial u}{\partial y}-\omega=g_{2} \text { in } \Omega
$$

$$
\frac{\partial \omega}{\partial y}+\frac{\partial p}{\partial x}=f_{1} \quad \text { in } \Omega
$$

and

$$
-\frac{\partial \omega}{\partial x}+\frac{\partial p}{\partial y}=f_{2} \text { in } \Omega
$$

where $g_{1}, g_{2}, f_{1}$, and $f_{2}$ are given functions. We consider the two sets of boundary conditions

$$
u=U \text { and } v=V \text { on } \Gamma \text { and } p(0,0)=P_{0}
$$

and

$$
p=P \text { and } u n_{1}+v n_{2}=W \text { on } \Gamma
$$

where $U, V, P$, and $W$ are given functions defined on $\Gamma, P_{0}$ is a given number, and $n_{1}, n_{2}$ denote the components of the unit outer normal. We will define the various data functions by choosing an exact solution $(u, v, \omega, p)$ and then substituting into the above equations.

We will measure the differences

$\left(L^{2}\right.$ error $)$

$$
\left\|\xi-\xi^{h}\right\|_{0}=\left(\int_{\Omega}\left(\xi-\xi^{h}\right)^{2} d \Omega\right)^{1 / 2}
$$

and

( $H^{1}$ error)

$$
\left|\xi-\xi^{h}\right|_{1}=\left(\int_{\Omega}\left[\left(\frac{\partial}{\partial x}\left(\xi-\xi^{h}\right)\right)^{2}+\left(\frac{\partial}{\partial y}\left(\xi-\xi^{h}\right)\right)^{2}\right] d \Omega\right)^{1 / 2}
$$

where $\xi$ could be any of $u, v, \omega$, or $p$.

Throughout we use piecewise quadratic finite element spaces; thus, for sufficiently smooth solutions and for the domain $\Omega=\{0 \leq x \leq 1,0 \leq y \leq 1\}$, we expect that for the boundary conditions (BC2) we have that

$$
\left\|\xi-\xi^{h}\right\|_{0}=O\left(h^{3}\right) \quad \text { and } \quad\left|\xi-\xi^{h}\right|_{1}=O\left(h^{2}\right)
$$


where again $\xi$ could be any of $u, v, \omega$, or $p$. This is confirmed by the computations that follow. Of special interest to us here is the computationally determined rates of converge for the boundary conditions (BC1).

The first example we present has the smooth exact solution

$$
\begin{aligned}
& u=-\cos \pi x \sin \pi y+1-y^{3}, \quad v=\sin \pi x \cos \pi y+1-x^{3}, \\
& \omega=\frac{\partial v}{\partial x}-\frac{\partial u}{\partial y}, \quad \text { and } \quad p=\sin y \cos x+x y^{2} .
\end{aligned}
$$

Figures 1 and 2 give $\log$-log plots of the $L^{2}$ and $H^{1}$ errors vs. the number of grid intervals in each direction for a uniform grid spacing. (Similar results have been obtained for nonuniform grid spacings.) From these figures one can confirm that for the boundary conditions (BC2) one does indeed obtain the best approximation convergence rates of (4.5). (As is usually the case, computed $L^{2}$ rates are usually less reliable than their $H^{1}$ counterparts.) In fact, the asymptotic slopes of each of the dotted curves of Figure 1 are approximately 3 while those of Figure 2 are approximately 2. Surprisingly, the slopes of the solid curves are asymptotically the same as those of the dotted curves; thus, for the boundary conditions (BC1) we seemingly obtain the best approximation rates of convergence of (4.5). Experiments with other smooth solutions yield similar results.
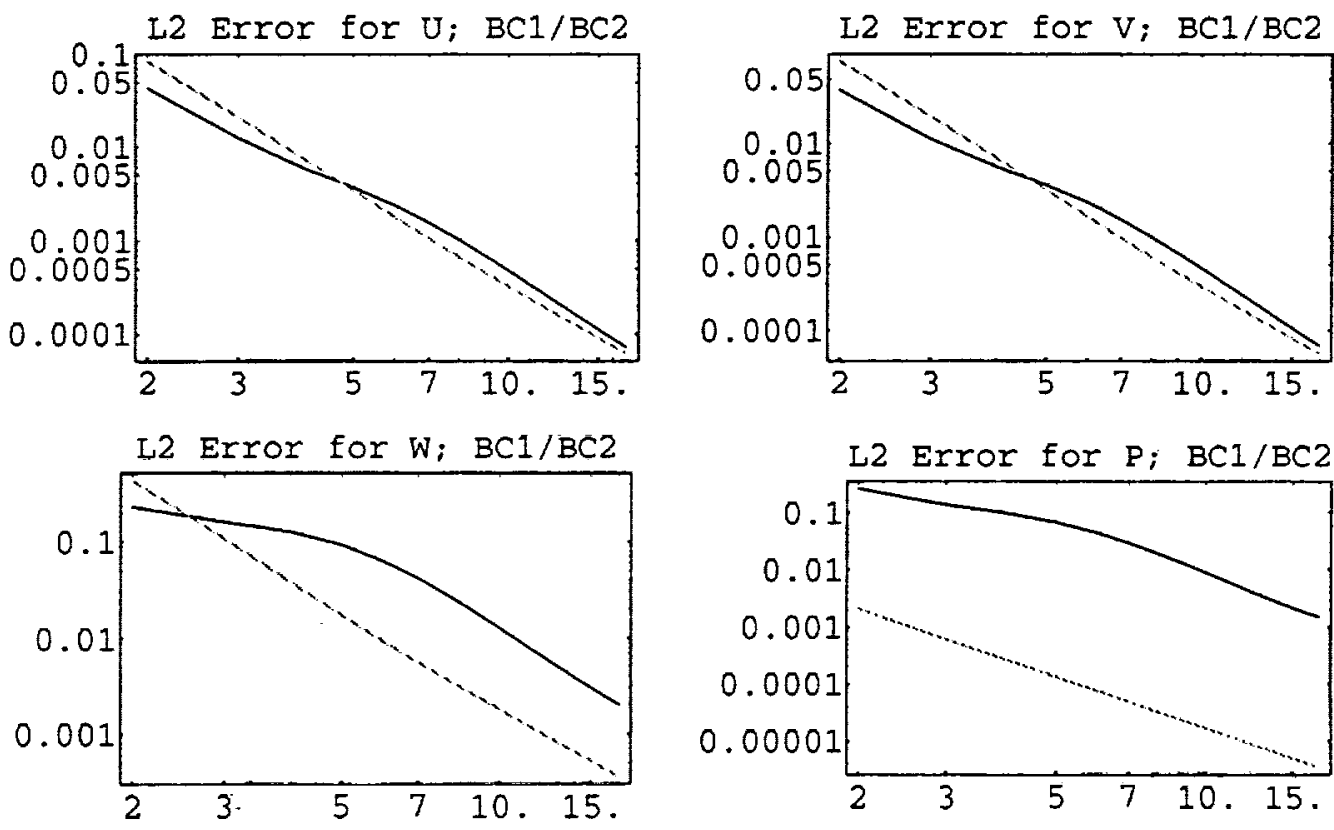

L2 Error for $\mathrm{P} ; \mathrm{BCl} / \mathrm{BC} 2$

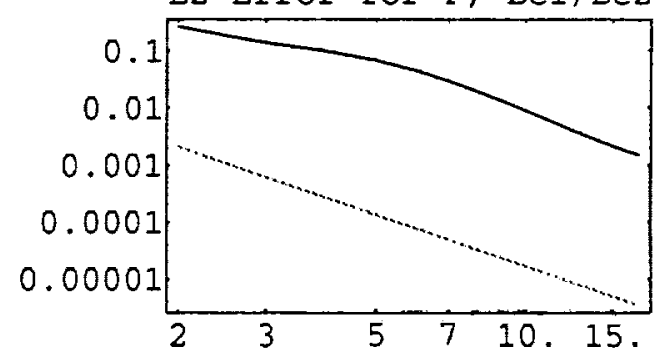

Fig. 1. $L^{2}$ errors vs. number of grid intervals in each direction for example (4.5). Solid line if for $\mathrm{BC} 1$; dotted curve is for $\mathrm{BC} 2$. 
H1 Error for U; $\mathrm{BC1} / \mathrm{BC2}$

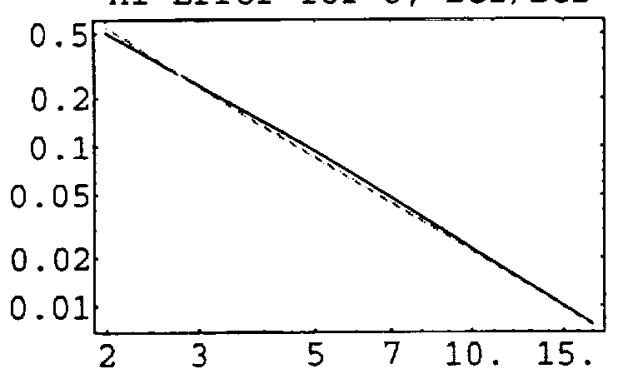

$\mathrm{H} 1$ Error for $\mathrm{W}$; $\mathrm{BC} 1 / \mathrm{BC} 2$

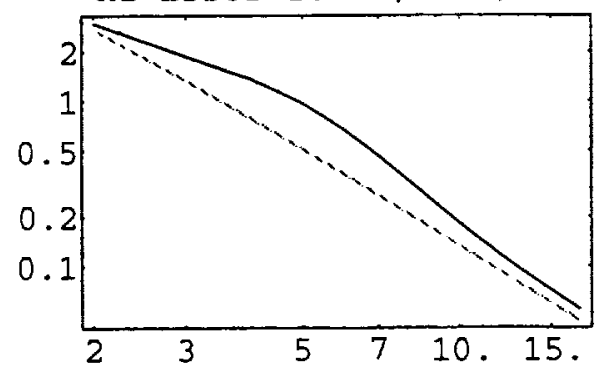

H1 Error for $V ; \quad B C 1 / B C 2$

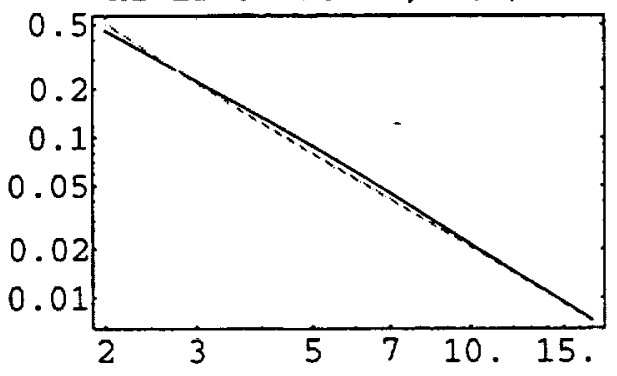

$\mathrm{H} 1$ Error for $\mathrm{P} ; \mathrm{BC} 1 / \mathrm{BC} 2$

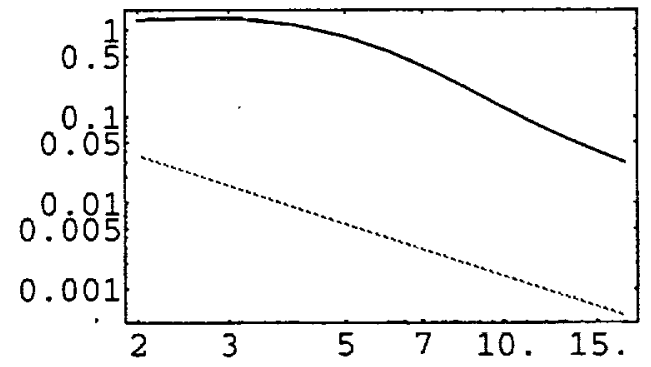

Fig. 2. $H^{1}$ errors vs. number of grid intervals in each direction for example (4.5). Solid line if for $\mathrm{BC1}$; dotted curve is for $\mathrm{BC2}$.

The next set of examples uses the exact solution

$$
u=v=\omega=p=\left((x-a)^{2}+(y-b)^{2}\right)^{s / 2}
$$

We use $a=b=0.1234$. The exponent $s$ may be chosen to adjust the smoothness of the exact solution, and thus the rates of convergence of the best approximations out of the finite element space used. Thus, except for some exceptional integer values, we have that

$$
\left\|\xi-\hat{\xi}^{h}\right\|_{0}=O\left(h^{t+1}\right) \quad \text { and } \quad\left|\xi-\hat{\xi}^{h}\right|_{1}=O\left(h^{t}\right), \quad \text { where } t=\min (s, 2)
$$

and where again $\xi$ denotes any of $u, v, \omega$, or $p$, and $\xi^{h}$ denotes the corresponding best approximation.

Figures 3-10 give log-log plots of $L^{2}$ and $H^{1}$ errors vs. the number of grid intervals in each direction for a uniform grid spacing and for different values of $s$. According to (4.7), the best approximation for the cases $s=2.5$ and $s=2.001$ have $L^{2}$ and $H^{1}$ errors of $O\left(h^{3}\right)$ and $O\left(h^{2}\right)$, respectively. Figures 3-6 show that the least-squares finite element solutions for both boundary conditions $(\mathrm{BC} 1)$ and $(\mathrm{BC} 2)$ also seem to converge at approximately this rate for these values of $s$. Similar conclusions can be drawn from Figures 7 and 8 for which the best approximations and both finite element solutions have $L^{2}$ and $H^{1}$ errors close to $O\left(h^{2.5}\right)$ and $O\left(h^{1.5}\right)$, respectively. Likewise, from Figures 9 and 10 we have that finite element solutions have $L^{2}$ and $H^{1}$ errors close to $O\left(h^{2}\right)$ and $O\left(h^{1}\right)$, respectively; these rates are again those of the best approximation for $s=1.001$. 

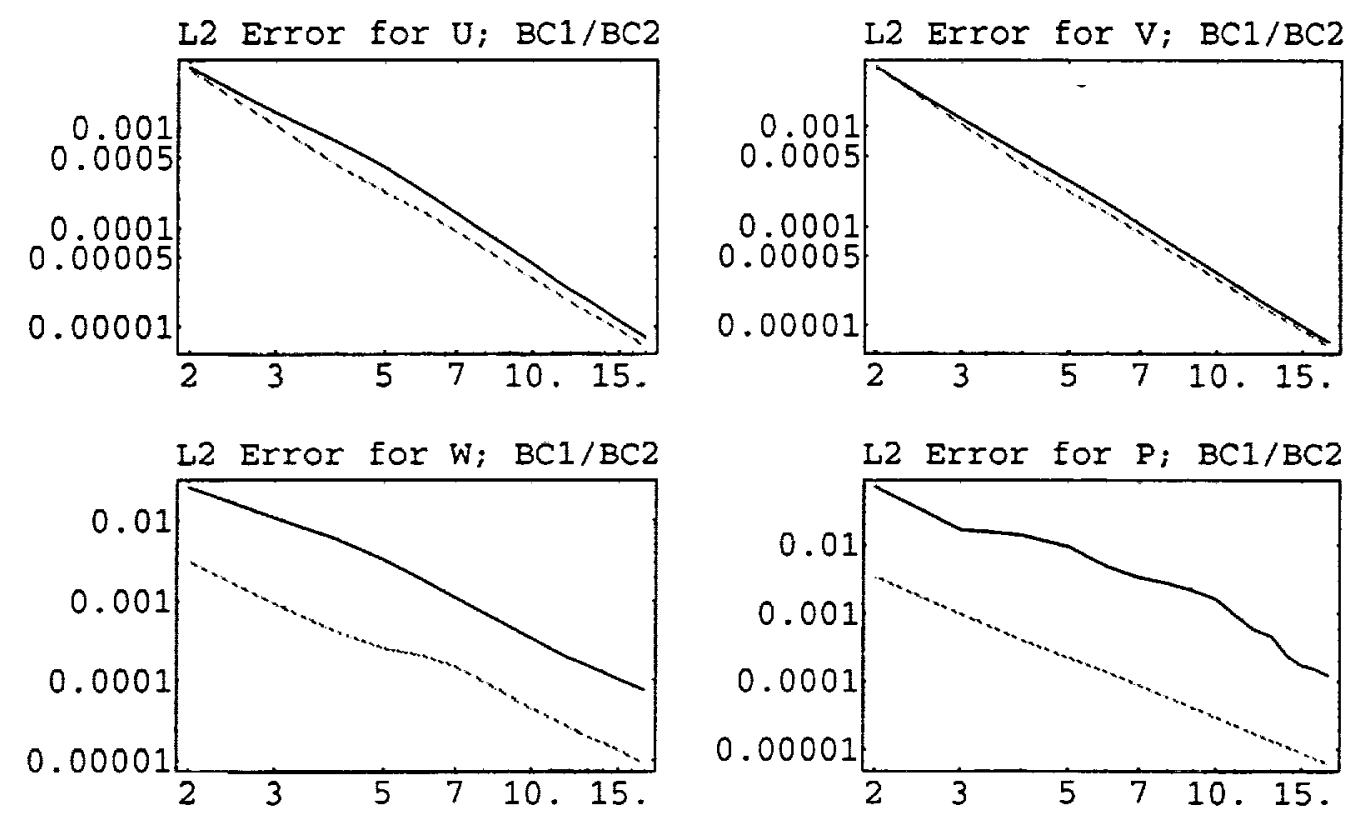

Fig. 3. $L^{2}$ errors vs. number of grid intervals in each direction for example (4.6) with $s=2.5$ Solid line if for $\mathrm{BC} 1$; dotted line is for $\mathrm{BC2}$.
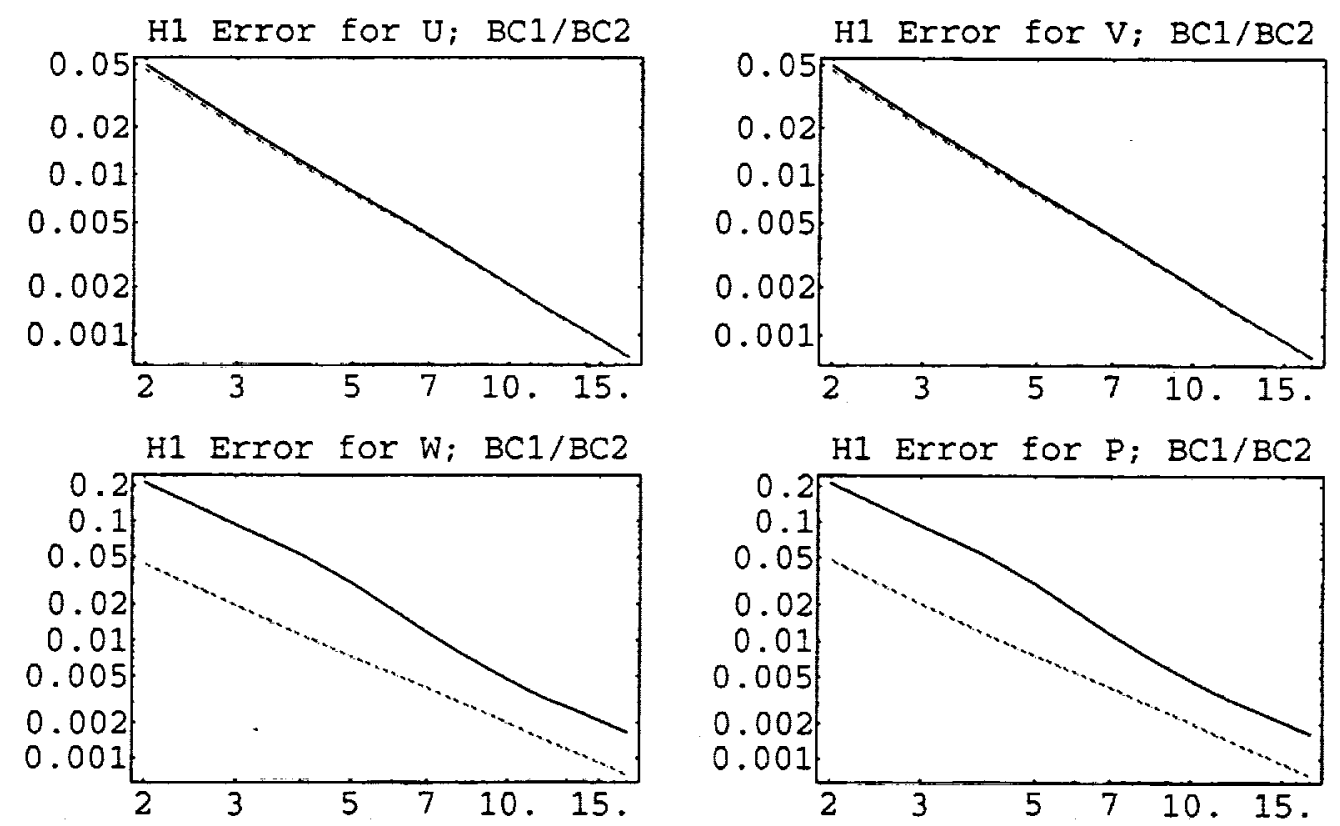

Fig. 4. $H^{1}$ errors us. number of grid intervals in each direction for example (4.6) with $s=2.5$ Solid line if for $\mathrm{BC} 1$; dotted line is for $\mathrm{BC} 2$. 
L2 Error for $\mathrm{U} ; \mathrm{BCl} / \mathrm{BC} 2$
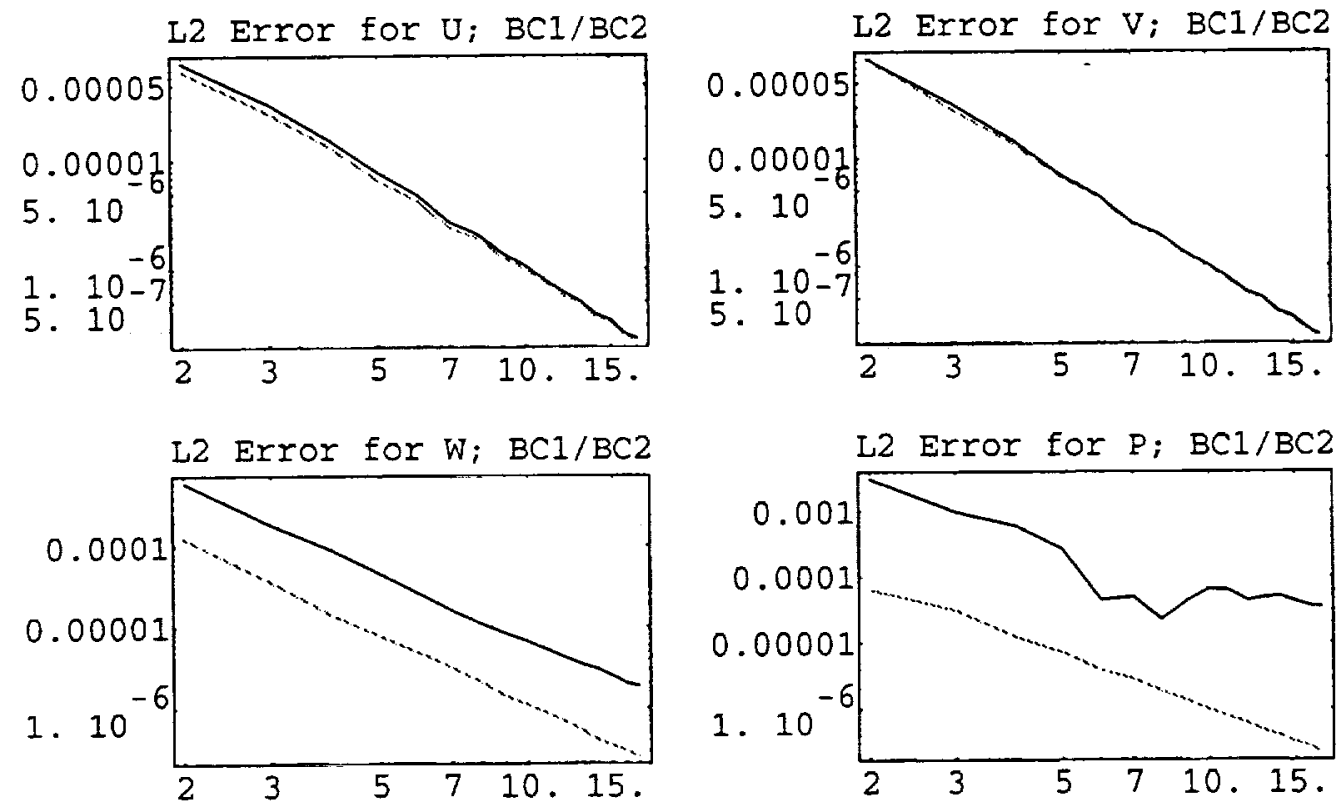

Fig. 5. $L^{2}$ errors us. number of grid intervals in each direction for example (4.6) with $s=2.01$ Solid line if for $\mathrm{BC1}$; dotted line is for $\mathrm{BC2}$.
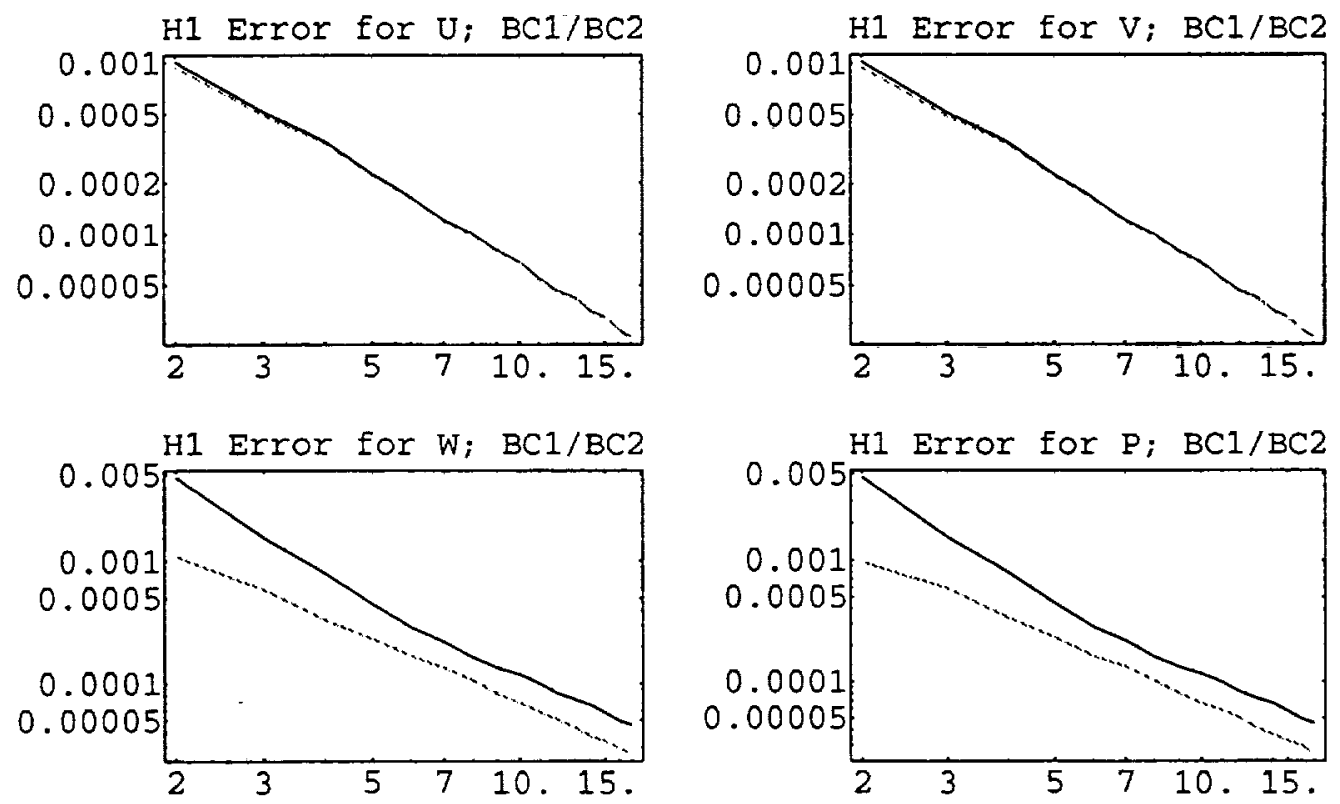

Fig. 6. $H^{1}$ errors vs. number of grid intervals in each direction for example (4.6) with $s=2.01$ Solid line if for $\mathrm{BCl}$; dotted line is for $\mathrm{BC2}$. 

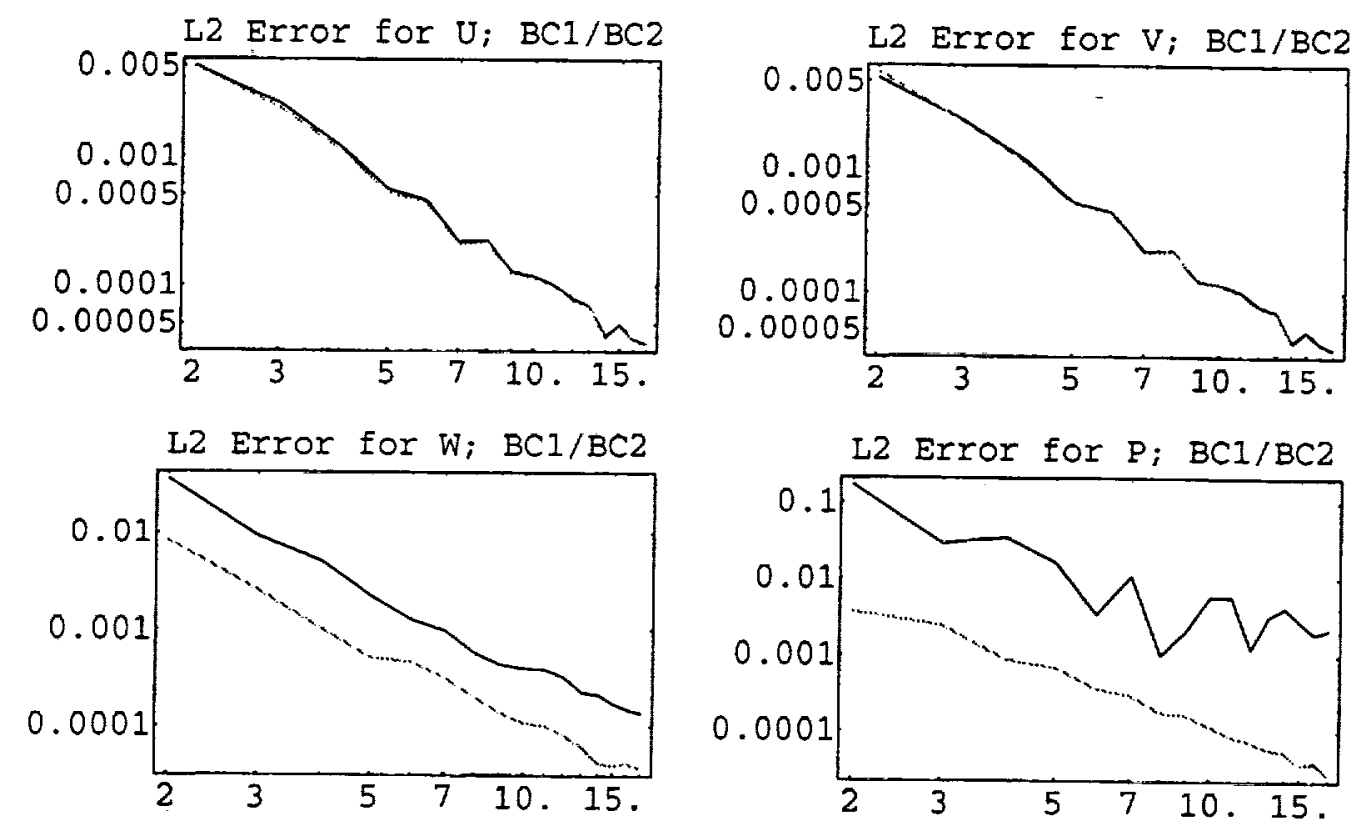

Fig. 7. $L^{2}$ errors vs. number of grid intervals in each direction for example (4.6) with $s=1.5$ Solid line if for $\mathrm{BC1}$; dotted line is for $\mathrm{BC2}$.
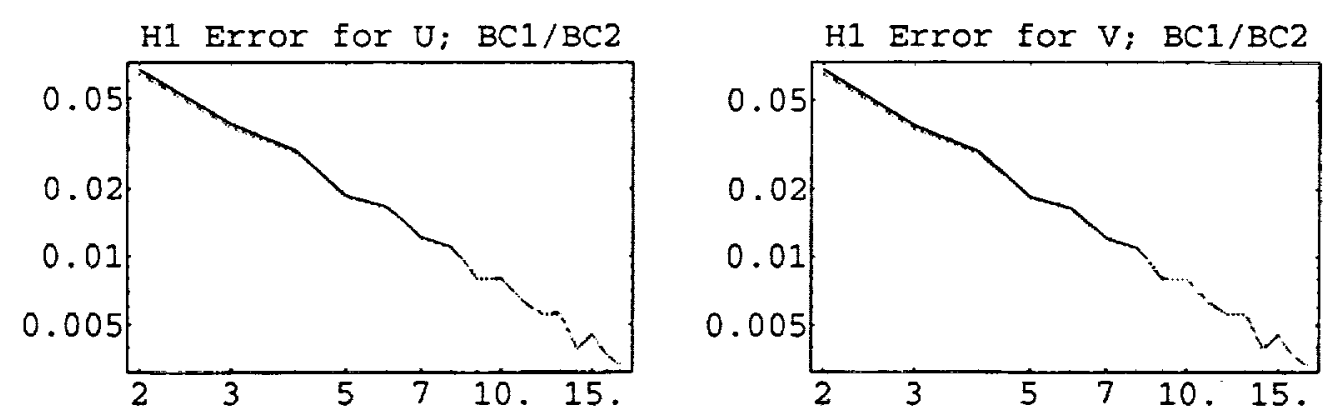

$\mathrm{H} 1$ Error for $\mathrm{W}$; $\mathrm{BC} 1 / \mathrm{BC} 2$
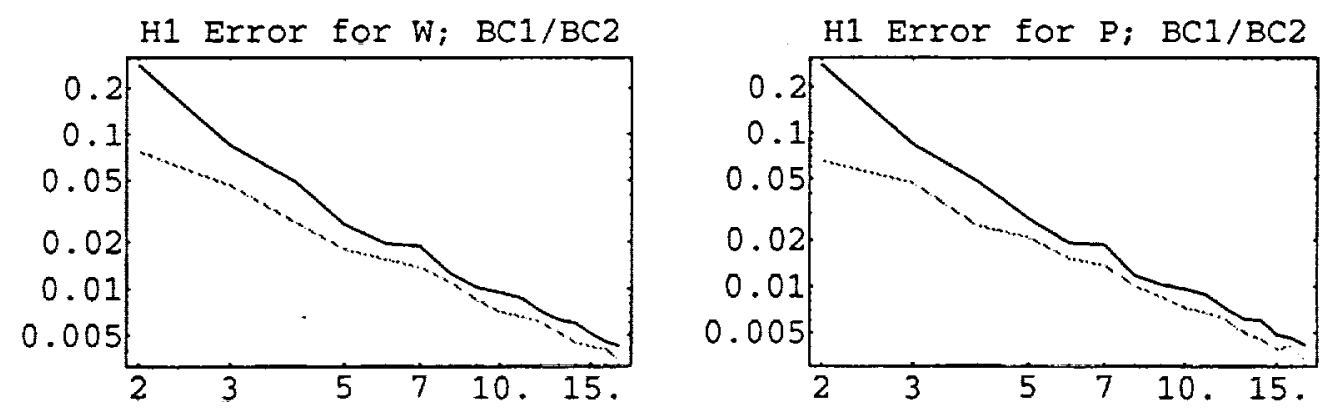

Fig. 8. $H^{1}$ errors $v s$. number of grid intervals in each direction for example (4.6) with $s=1.5$ Solid line if for $\mathrm{BC} 1$; dotted line is for $\mathrm{BC2}$. 

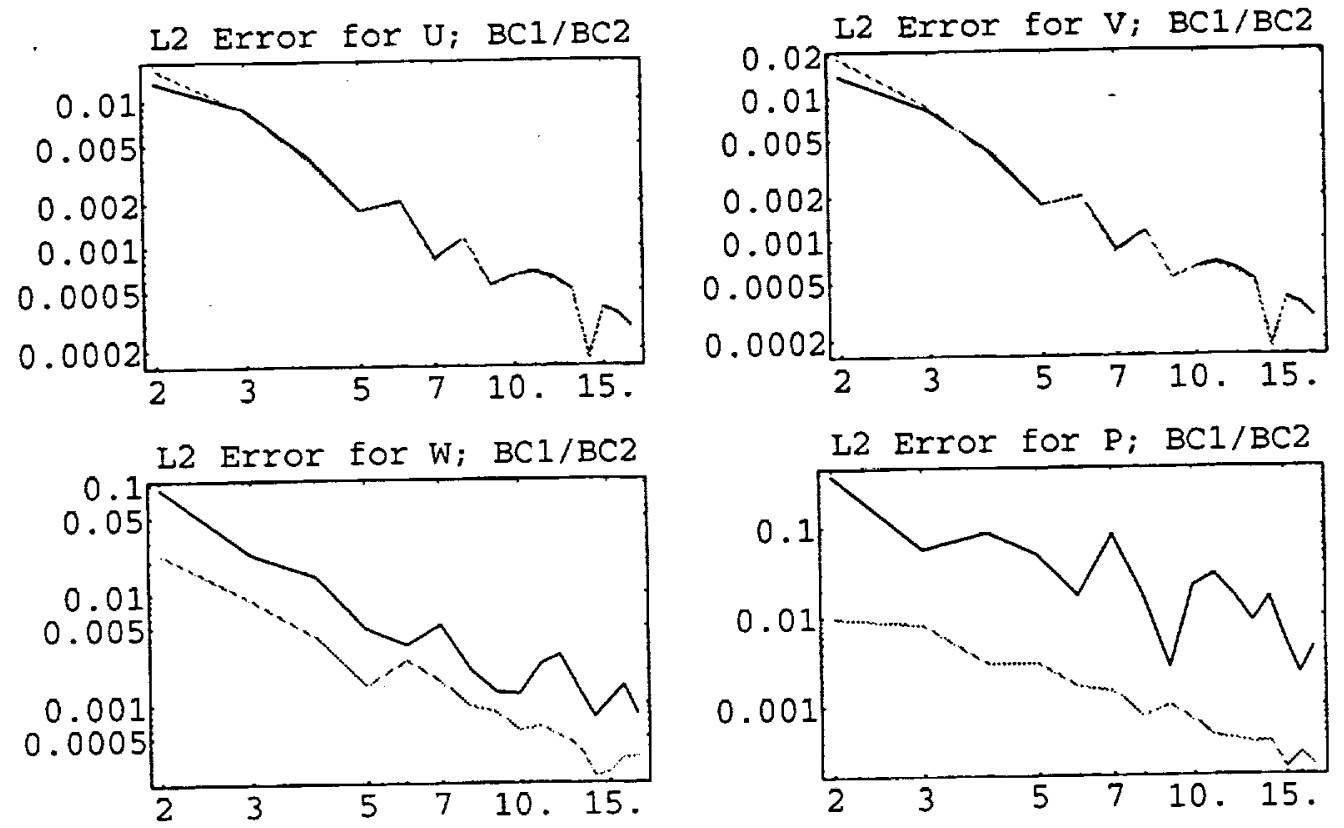

Fig. 9. $L^{2}$ errors vs. number of grid intervals in each direction for example (4.6) with $s=1.001$ Solid line if for $\mathrm{BC} 1$; dotted line is for $\mathrm{BC}$.
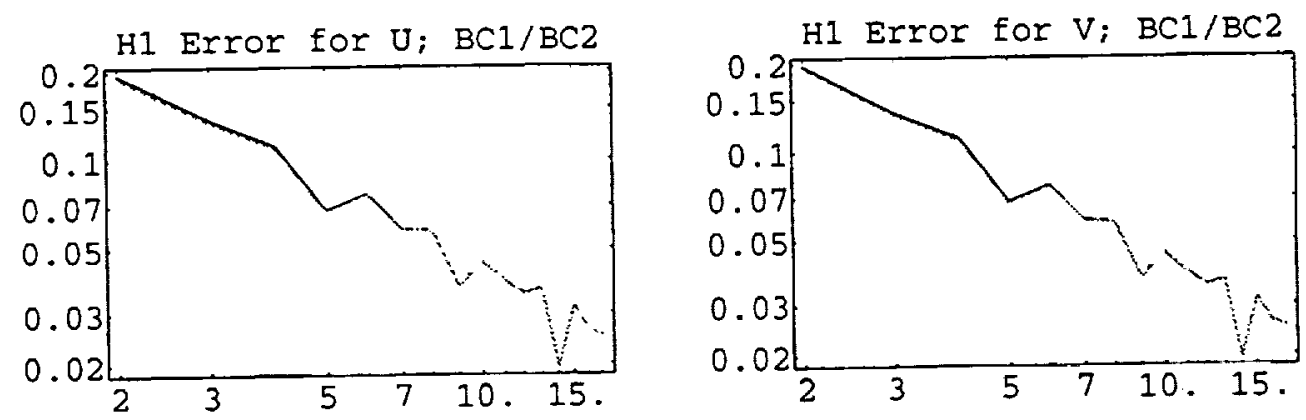

$\mathrm{H} 1$ Error for $W$; $B C 1 / B C 2$

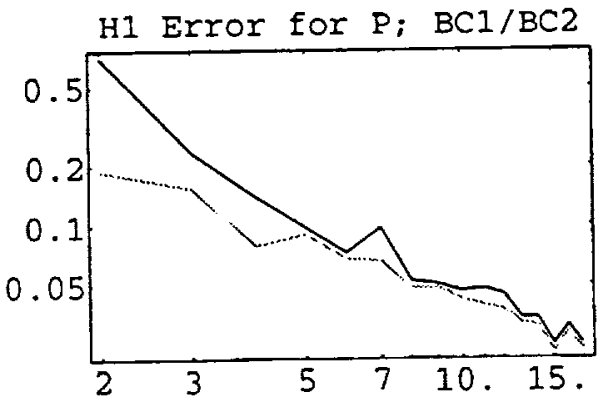

Fig. 10. $H^{1}$ errors vs. number of grid intervals in each direction for example (4.6) with $s=1.001$ Solid line if for $\mathrm{BC1}$; dotted line is for $\mathrm{BC2}$. 
The above conclusions drawn from Figures 1-10 can be quantized by computing the slope of a least-squares straight line fit to the various curves in the figure. The results are given in the following table. The first column of the table gives the exact solution used to obtain the remaining columns. The second column gives the error type, i.e., either $H^{1}$ or $L^{2}$, to which the entries of the remaining columns correspond. The third column gives the rate of convergence of the best approximations to the exact solutions out of the quadratic finite element space. Specifically, that column gives the value of $t$ for the $H^{1}$ error and $t+1$ for the $L^{2}$ error as determined from (4.7) The fourth column gives the exponent $\beta$ in the error formula

$$
\left\|u-u^{h}\right\|=O\left(h^{\beta}\right),
$$

where $\|\cdot\|$ denotes either the $L^{2}$ norm or the $H^{1}$ semi-norm, $u$ the exact x-component of the velocity, and $u^{h}$ its least-squares finite element approximation. The exponent $\beta$ is determined computationally from the data that was used to produce the plots for the errors in the approximation to $u$ in Figures 1-10. Specifically, $\beta$ was determined as the slope of the best least-squares straight line fit of the curves in those figures. The remaining three columns give similar data for $v, \omega$, and $p$.

\begin{tabular}{|c|c|c|c|c|c|c|c|c|}
\hline \multicolumn{2}{|c|}{ Exact solution } & \multirow[t]{2}{*}{ BC type } & \multirow[t]{2}{*}{ Error type } & \multicolumn{5}{|c|}{ Rates of convergence } \\
\hline & & & & b.a. & $u$ & $v$ & $\omega$ & $p$ \\
\hline & $(4.5)$ & $\begin{array}{l}\mathrm{BC} 1 \\
\mathrm{BC} 2\end{array}$ & $\begin{array}{l}H^{1} \\
L^{2} \\
H^{1} \\
L^{2}\end{array}$ & $\begin{array}{l}2 \\
3 \\
2 \\
3\end{array}$ & $\begin{array}{l}1.97 \\
3.54 \\
1.98 \\
3.10\end{array}$ & $\begin{array}{l}1.96 \\
3.61 \\
1.98 \\
3.11\end{array}$ & $\begin{array}{l}2.01 \\
3.44 \\
1.98 \\
3.04\end{array}$ & $\begin{array}{l}2.09 \\
3.21 \\
2.00 \\
3.00\end{array}$ \\
\hline (4.6) & $s=2.5$ & $\begin{array}{l}\mathrm{BC} 1 \\
\mathrm{BC} 2\end{array}$ & $\begin{array}{c}H^{1} \\
L^{2} \\
H^{1} \\
L^{2}\end{array}$ & $\begin{array}{l}2 \\
3 \\
2 \\
3\end{array}$ & $\begin{array}{l}1.93 \\
3.00 \\
1.93 \\
2.96\end{array}$ & $\begin{array}{l}1.93 \\
2.98 \\
1.93 \\
2.97\end{array}$ & $\begin{array}{l}1.88 \\
2.88 \\
1.90 \\
2.89\end{array}$ & $\begin{array}{l}1.89 \\
2.92 \\
1.94 \\
2.94\end{array}$ \\
\hline$(4.6)$ & $s=2.01$ & $\begin{array}{l}\mathrm{BC} 1 \\
\mathrm{BC} 2\end{array}$ & $\begin{array}{c}H^{1} \\
L^{2} \\
H^{1} \\
L^{2}\end{array}$ & $\begin{array}{l}2 \\
3 \\
2 \\
3\end{array}$ & $\begin{array}{l}1.98 \\
2.82 \\
1.98 \\
2.93\end{array}$ & $\begin{array}{l}1.98 \\
2.81 \\
1.98 \\
2.95\end{array}$ & $\begin{array}{l}1.86 \\
2.70 \\
1.84 \\
2.71\end{array}$ & $\begin{array}{l}1.85 \\
2.98 \\
1.76 \\
2.87\end{array}$ \\
\hline$(4.6)$ & $s=1.5$ & $\begin{array}{l}\mathrm{BC} 1 \\
\mathrm{BC} 2\end{array}$ & $\begin{array}{c}H^{1} \\
L^{2} \\
H^{1} \\
L^{2}\end{array}$ & $\begin{array}{l}1.5 \\
2.5 \\
1.5 \\
2.5\end{array}$ & $\begin{array}{l}1.41 \\
2.39 \\
1.39 \\
2.39\end{array}$ & $\begin{array}{l}1.41 \\
2.42 \\
1.39 \\
2.42\end{array}$ & $\begin{array}{l}1.82 \\
2.52 \\
1.43 \\
2.52\end{array}$ & $\begin{array}{l}1.82 \\
2.36 \\
1.41 \\
2.36\end{array}$ \\
\hline$(4.6)$ & $s=1.001$ & $\begin{array}{l}\mathrm{BC} 1 \\
\mathrm{BC} 2\end{array}$ & $\begin{array}{c}H^{1} \\
L^{2} \\
H^{1} \\
L^{2}\end{array}$ & $\begin{array}{l}1.001 \\
2.001 \\
1.001 \\
2.001\end{array}$ & $\begin{array}{l}0.99 \\
1.87 \\
0.98 \\
1.92\end{array}$ & $\begin{array}{l}0.99 \\
1.87 \\
0.98 \\
1.94\end{array}$ & $\begin{array}{l}1.34 \\
1.98 \\
0.97 \\
2.06\end{array}$ & $\begin{array}{l}1.37 \\
1.81 \\
0.97 \\
1.89\end{array}$ \\
\hline
\end{tabular}

Table 1. Rates of convergence of the $H^{1}$ and $L^{2}$ errors in the best approximation (b.a.) and in the least-squares finite element solution $(u, v, \omega, p)$. 
Note that the behavior of the $L^{2}$ errors is much more erratic than that of the $H^{1}$ errors; this is usually the case. However, we see that the errors for $(\mathrm{BC} 1)$ are not all that different from those for (BC2) and we may conclude that the errors in the former case are, at the least, nearly optimal.

\section{CONCLUDING REMARKS}

We have studied the accuracy of a least-squares finite element method for the Navier-Stokes equations based on a velocity-vorticity-pressure formulation. In particular, we have focused on velocity boundary conditions. In this case standard techniques for error estimation fail so that a computational study of accuracy is called for. In spite of this failure, the computational experiments reported on here indicate that the accuracy is, at the least, nearly optimal, i.e., that the rate of convergence as the grid size is reduced is seemingly the same as that for the best approximations out of the finite element spaces. When the least-squares finite element discretization algorithm is coupled with a Newton linearization with continuation, desirable discrete algebraic properties result. Thus, the overall method seems to provide a good combination of accuracy and efficiency.

There remains substantial issues to study connected with the least-squares finite element method for incompressible flows. These include practical implementation issues such as the use of iterative linear solvers and theoretical issues such as the derivation of rigorous error estimates. We will address these issues in a forthooming paper.

\section{REFERENCES}

[1] Agmon, S., A. Douglis, and L. Niremberg, Estimates near the boundary for solutions of elliptic partial differentia] equations satisfying general boundary conditions I, II, Comm. Pure Appl. Math. 12 1959, 623-727; 17 1964, 35-92.

[2] Brezzi, F., J. Rappaz, and P.-A. Raviart, Finite-dimensional approximation of nonlinear problems, Part I: Branches of nonsingular solutions, Numer. Math. 36 1980, 1-25.

[3] Chang, C., Least squares finite-element method for incompressible flow in 3-D, to appear.

[4] Chang, C., Piecewise linear approach to the Stokes equations in 3-D, to appear.

[5] Chang, C., and B.-N. Jiang, An error analysis of least-squares finite element methods of velocity-pressurevorticity formulation for the Stokes problem, Comp. Meth. Appl. Mech. Engrg. 84 1990, 247-255.

[6] Crouzeix, M., and J. Rappaz, On Numerical Approximation in Bifurcation Theory, Springer, Berlin, 1990.

[7] Franca, L., T. Hughes, and R. Stenberg, Stabilized finite elements, Incampressible Computational Fluid Dynamics: Trends and Advances, (Ed. by M. Gunzburger and R. Nicolaides), Cambridge, to appear.

[8] Fix, G., M. Gunzburger, and R. Nicolaides, On finite element methods of the least squares type, Comput. Math. Appl. 5 1979, 87-98.

[9] Girault, V. and P.-A. Raviart, Finite Element Methods for Navier-Stokes Equations, Springer, Berlin, 1986.

[10] Gunzburger, M., Finite Element Methods for Viscous Incompressible Flous, Academic, Baston, 1989.

[11] Gunzburger, M. and R. Nicolaides, Incompressible Computational Fluid Dynamics: Trends and Advances, Cambridge, to appear.

[12] Gunzburger, M. and J. Peterson, Predictor and steplength selection in continuation methods for the NavierStokes equations, Comput. Math. Appl. 22 1991, 72-81.

[13] Jiang, B.-N., A least-squares finite element method for the incompressible Navier-Stokes equations, Int. J. Numer. Meth. Fluids 14 1992, 843-859. 
[14] Jiang, B.-N., and C. Chang, Least-squares finite elements for the Stokes problem, Comp. Meth. Appl. Mech. Engrg. 78 1990, 297-311.

[15] Jiang, B.-N., T. Lin, and L. Povinelli, Large-scale computation of incompressible viscous flow by least-squares finite element method, to appear.

[16] Jiang, B.-N., and L. Povinelli, Least-squares finite element method for fluid dynamics, Comp. Meth. Appl. Mech. Engrg. 81 1990, 13-37.

[17] Jiang, B.-N., and V. Sonnad, Least-squares solution of incompressible Navier-Stokes equations with the p-version of finite elements, to appear.

[18] Keller, H., Numerical Methods in Bifurcation Problems, Springer, Berlin, 1987.

(19) Keller, H., Global homotopies and Newton methods, Recent Advances in Numerical Analysis (Ed. by C. de Boor and G. Golub), Academic, New York, 1978, 73-79.

[20] Lefebvre, D., J. Peraire, and K. Morgan, Least squares finite element solution of compressible and incompressible Hows, to appear.

[21] Rheinboldt, W., Solution fields of nonlinear equations and continuation methods, SIAM J. Numer. Anal. 17 1980, 221-237.

[22] Tang, L. and T. Tsang, A least-squares finite element for time-dependent incompressible flows with thermal convection, to appear.

[23] Wedland, W., Elliptic Systems in the Plane, Pittman, London, 1979. 
Public reporting burden for this collection of information is estimated to average 1 hour per response, including the time for reviewing instructions, searching existing data sources, gatheing and maintaining the collection of information. Send comments regarding this burden estimate or any other aspect of this

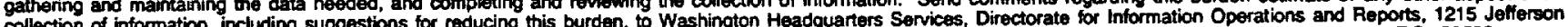
Davis Highway, Sulte 1204, Arlington, VA 22202-4302, and to the Office of Management and Budget, Paperwork Reduction Project (0704-0188), Washington, DC 20503.
1. AGENCY USE ONLY (Leave blank)
2. REPORT DATE
June 1993
3. REPORT TYPE AND DATES COVERED
Technical Memorandum

4. TIILE AND SUBTIIE

Accuracy of Least-Squares Methods For The Navier-Stokes Equations

6. AUTHOR(S)

WU-505-90-5K

Pavel B. Bochev and Max D. Gunzburger

7. PERFORMING ORGANRATION NAME(S) AND ADDRESS(ES)

National Aeronautics and Space Administration

Lewis Research Center

5. FUNDING NUMBEAS

Cleveland, Ohio 44135-3191

8. PERFORUING ORGANIZATION REPORT NUMBER

E-7919

9. SPONSORINGMONITORING AGENCY NAME(S) AND ADDRESS(ES)

10. SPONSORINGMONITORING AGENCY REPORT NUMBER

National Aeronautics and Space Administration

Washington, D.C. 20546-0001

NASA TM-106209

ICOMP-93-19

11. SUPPLEMENTARY NOTES

Pavel B. Bohev, Virginia Polytechnic Institute and State University, Department of Mathematics and Interdisciplinary Center for Applied Mathematics, Blacksburg, Virginia 24061 and Max D. Gunzburger, Institute for Computational Mechanics in Propulsion, NASA Lewis Research Center, (work funded under NASA Cooperative Agreement NCC3-233) and Virginia Polytechnic Institute and State University, Blacksburg, Virginia 24061. ICOMP Program Director, Lovis A. Povinelli, (2316) 433-5818.

12a. DISTRIBUTIONJAVAILABILITY STATEMENT

12b. DISTRIBUTION CODE

Unclassified-Unlimited

Subject Category 34

\section{ABSTRACT (Maximum 200 words)}

Recently there has been substantial interest in least-squares finite element methods for velocity-vorticity-pressure formulations of the incompressible Navier-Stokes equations. The main cause for this interest is the fact that algorithms for the resulting discrete equations can be devised which require the solution of only symmetric, positive definite systems of algebraic equations. On the other hand, it is well-documented that methods using the vorticity as a primary variable often yield very poor approximations. Thus, here we study the accuracy of these methods through a series of computational experiments, and also comment on theoretical error estimates. It is found, despite the failure of standard methods for deriving error estimates, that computational evidence suggests that these methods are, at the least, nearly optimally accurate. Thus, in addition to the desirable matrix properties yielded by least-squares methods, one also obtains accurate approximations.

\section{SUBJECT TERMS}

Incompressible computational fluid dynamis; Least squares methods; Finite element methods

\begin{tabular}{|c|c|}
\hline 17. SECUATY CLASSIFICATION & $\begin{array}{c}\text { 18. SECURTY CLASSIFICATION } \\
\text { OF REPORT } \\
\text { OF THIS PAGE } \\
\text { Unclassified }\end{array}$ \\
\hline
\end{tabular}

19. SECURTY CLASSIFICATION OF ABSTRACT Unclassified 
